\title{
TU/e EmonOWEN

\section{UV detection in capillary zone electrophoresis : peaks or dips - that is the question}

Citation for published version (APA):

Beckers, J. L. (1994). UV detection in capillary zone electrophoresis : peaks or dips - that is the question. Journal of Chromatography, A, 679(1), 153-165. https://doi.org/10.1016/0021-9673(94)80322-6

DOI:

10.1016/0021-9673(94)80322-6

Document status and date:

Published: 01/01/1994

\section{Document Version:}

Publisher's PDF, also known as Version of Record (includes final page, issue and volume numbers)

\section{Please check the document version of this publication:}

- A submitted manuscript is the version of the article upon submission and before peer-review. There can be important differences between the submitted version and the official published version of record. People interested in the research are advised to contact the author for the final version of the publication, or visit the $\mathrm{DOI}$ to the publisher's website.

- The final author version and the galley proof are versions of the publication after peer review.

- The final published version features the final layout of the paper including the volume, issue and page numbers.

Link to publication

\section{General rights}

Copyright and moral rights for the publications made accessible in the public portal are retained by the authors and/or other copyright owners and it is a condition of accessing publications that users recognise and abide by the legal requirements associated with these rights.

- Users may download and print one copy of any publication from the public portal for the purpose of private study or research.

- You may not further distribute the material or use it for any profit-making activity or commercial gain

- You may freely distribute the URL identifying the publication in the public portal.

If the publication is distributed under the terms of Article 25fa of the Dutch Copyright Act, indicated by the "Taverne" license above, please follow below link for the End User Agreement:

www.tue.nl/taverne

Take down policy

If you believe that this document breaches copyright please contact us at:

openaccess@tue.nl

providing details and we will investigate your claim. 


\title{
UV detection in capillary zone electrophoresis Peaks or dips - that is the question
}

\author{
J.L. Beckers \\ Laboratory of Instrumental Analysis, Eindhoven University of Technology, P.O. Box 513, 5600 MB Eindhoven, Netherlands
}

First received 30 March 1994; revised manuscript received 27 May 1994

\begin{abstract}
Applying capillary zone electrophoresis with UV detection, both UV-absorbing and UV-transparent components can be present in electropherograms as negative peaks (dips) or as positive peaks. Starting from Kohlrausch's regulation function, derived for fully ionized monovalent ionic constituents and under the assumption that the molar absorptivities of the UV-absorbing components are identical, eight different cases can be distinguished and in several cases components can occur both as peaks or as dips depending on their mobilities and those of the co-ions of the system. Applying background electrolytes containing two co-ions, system peaks are present, with a mobility that is between the mobilities of the two co-ions and determined by the concentration ratio of these two co-ions. In the background electrolytes studied, containing the co-ions potassium and histidine, UV-transparent sample components with a mobility higher than that of the system peak migrate as a positive peak, whereas UVtransparent components with lower mobilities migrate as negative peaks. System peaks themselves can also be pcaks or dips depending on the sample composition. Sample peaks in the vicinity of system peaks interact with the system peaks through which both sample and system peaks are enlarged and quantitative properties are lost. Similar phenomena can be measured for anions in background electrolytes containing the co-ions phenylacetate and acetate, indicating that these phenomena are probably not associated with adsorption phenomena of cations on the fused-silica surface.
\end{abstract}

\section{Introduction}

Most commercially available capillary zone electrophoresis (CZE) apparatus is equipped with selective detectors, such as UV and laserinduced fluorescence (LIF) detectors, and not with a universal detector, such as a conductivity detector. Applying selective detectors, e.g., a UV detector, UV-absorbing components can be measured in the "direct UV mode". UV-transparent components, however, often have to be detected in the indirect mode. With indirect detection, the analyte displaces a chromophore present in the background electrolyte (BGE), whereby the concentration of the limit of detection, $c_{\text {LOD }}$, can be determined by

$c_{\text {LOD }}=c_{\mathrm{M}} / R D_{\mathrm{r}}$

with $c_{M}$ is the concentration of the BGE constituent that is being monitored, $R$ is the response factor or transfer ratio and $D_{1}$ is the dynamic reserve, equal to the signal-to-noise ratio $(S / N)$ [1]. From Eq. 1, it could be concluded that a low $c_{\text {LOD }}$ can be obtained by applying low $c_{\mathrm{M}}$ values and a high $D_{r}[1-3]$, although in practice also $D_{\mathrm{r}}$ decreases at lower 
$c_{\mathrm{M}}$ values [4]. Nielen [5] calculated the response factor, dependent on the mobilities. Foret et al. [6] discussed that the highest sensitivity can be achieved for sample ions having an effective mobility close to the mobility of the absorbing co-ion. So far, less attention has been paid to the presence of more than one UV-absorbing component in CZE systems. In that case both UVabsorbing and UV-transparent components can be met as negative or as positive peaks, depending on their molar absorptivity and transfer ratio. In this paper we discuss the question "peaks or dips?", due to the presence of both UV-absorbing counter- and co-ions, and the effect of the presence of two co-ions in a BGE on system peaks in UV detection and the influence on calibration graphs.

\section{Theoretical}

Assuming only the presence of fully ionized monovalent ionic constituents, the electrophoretic separation mechanism can be approximately described by Kohlrausch's regulation function [7]:

$\sum_{i} \frac{c_{i}}{m_{i}}=\omega$

where $c_{i}$ and $m_{i}$ represent the ionic concentrations and absolute values of the effective mobilities of all ionic constituents and the numerical value of the Kohlrausch function $\omega$ is locally invariant in time [8]. If the separation compartment in CZE is filled with a background electrolyte $\Lambda \mathrm{C}$, consisting of a co-ion $\Lambda$ and a counter ion $\mathrm{C}$, for the relationship between the concentration of the co-ions in the $\mathrm{BGE}, c_{\mathrm{A}}^{\mathrm{BGE}}$, and in a sample zone $\mathrm{S}, c_{\mathrm{A}}^{\mathrm{S}}$, containing also the sample ions $i$, can be derived $[9,10]$ :

$c_{\mathrm{A}}^{\mathrm{BGE}}=c_{\mathrm{A}}^{\mathrm{S}}+c_{i}^{\mathrm{S}} k_{i}$

with

$k_{i}=\frac{m_{i}+m_{\mathrm{c}}}{m_{\mathrm{A}}+m_{\mathrm{c}}} \cdot \frac{m_{\mathrm{A}}}{m_{i}}$

The superscripts BGE and S refer to the compo- sition of the pure background electrolyte $\mathrm{AC}$ and the sample zone, respectively. The concentration of the counter ion $\mathrm{C}$ is determined by the electroneutrality condition.

The transfer ratio or response factor, often denoted as $R$, is defined as the number of molecules of the BGE displaced by each analyte molecule and is in fact equal to the constant $k_{i}$ in Eq. 4:

$R=\frac{c_{\mathrm{A}}^{\mathrm{BGE}}-c_{\mathrm{A}}^{\mathrm{s}}}{c_{i}^{\mathrm{s}}}=\frac{\left|\Delta c_{\Lambda}\right|}{c_{i}^{\mathrm{s}}}=k_{i}$

Applying a UV detector, the measured absorbance $A$ will be

$A=\epsilon c l$

where $\varepsilon$ is the molar absorptivity $(1 / \mathrm{mol} \cdot \mathrm{cm})$ and $l$ is the effective path length in the detector (cm). For the carrier electrolyte this means

$A^{\mathrm{BGE}}=\left(\epsilon_{\mathrm{A}}+\epsilon_{\mathrm{C}}\right) c_{\mathrm{A}}^{\mathrm{BGE}} l$

For a sample zone the absorbance will be

$A^{\mathrm{s}}=\left(\epsilon_{\mathrm{A}}+\epsilon_{\mathrm{C}}\right) c_{\mathrm{A}}^{\mathrm{s}} l+\left(\epsilon_{i}+\epsilon_{\mathrm{C}}\right) c_{i}^{\mathrm{s}} l$

For the UV signal of a sample zone, applying Eq. 3, we can derive

$$
\begin{aligned}
\mathrm{d} A & =A^{\mathrm{s}}-A^{\mathrm{BGE}}=c_{i}^{\mathrm{s}} l\left[\left(\epsilon_{i}+\epsilon_{\mathrm{C}}\right)-\left(\epsilon_{\mathrm{A}}+\epsilon_{\mathrm{C}}\right) k_{i}\right] \\
& =c_{i}^{\mathrm{s}} l\left[\epsilon_{i}-k_{i} \epsilon_{\mathrm{A}}-\left(k_{i}-1\right) \epsilon_{\mathrm{C}}\right]=c_{i}^{\mathrm{s}} l K_{i}
\end{aligned}
$$

If the bracketed term in Eq. 9 is indicated by $K_{i}$, the spatial peak area will be proportional to $K_{i} Q_{\text {inj }}$ and the measured peak area on a temporal basis to $K_{i} Q_{\text {inj }} t_{i}$, where $Q_{\text {inj }}$ is the injected amount and $t_{i}$ is the migration time of a sample ion $i[10]$.

If sample components have an equal concentration $c_{i}$ in their zones, the peak heights are proportional to $K_{i}$. Generally, the concentrations $c_{i}$ will not only be determined by the sample concentration but by several other factors such as the concentrating effect, electrodispersive effects and diffusion.

From Eq. 9, all possible situations in UV detection in CZE can be deduced. If for ease of survey components are considered to be UV transparent or UV absorbing with equal molar 
Table 1

All possible combinations of ( + ) UV-absorbing or (-) UV-transparent sample components $i$, co-ions $A$ and counter-jons $C$, the expressions of $K_{i}$ values according to Eq. 9 and values for $K_{i}$ for the UV modes and indirect UV modes assuming equal molar absorptivities $\epsilon$ for the UV-absorbing ionic species

\begin{tabular}{|c|c|c|c|c|c|c|}
\hline \multirow[t]{2}{*}{ No. } & \multicolumn{3}{|c|}{ Component } & \multirow{2}{*}{$\begin{array}{l}K_{i}: \\
\epsilon_{i}-k_{i} \epsilon_{\mathrm{A}}-\left(k_{i}-1\right) \epsilon_{\mathrm{C}}\end{array}$} & \multicolumn{2}{|l|}{$K_{i}$} \\
\hline & $i$ & A & $\mathrm{C}$ & & UV mode & Indirect UV mode \\
\hline 1 & + & + & + & $\epsilon_{i}-k_{i} \epsilon_{\mathrm{A}}-\left(k_{i}-1\right) \epsilon_{\mathrm{C}}$ & $\left(2-2 k_{i}\right) \epsilon$ & \\
\hline 2 & + & + & - & $\epsilon_{i}-k_{i} \epsilon_{\mathrm{A}}$ & $\left(1-k_{i}\right) \epsilon$ & \\
\hline 3 & + & - & + & $\epsilon_{i}-\left(k_{i}-1\right) \epsilon_{c}$ & $\left(2-k_{i}\right) \epsilon$ & \\
\hline 4 & + & - & - & & $\epsilon$ & \\
\hline 5 & - & + & + & $-k_{i} \epsilon_{\mathrm{A}}-\left(k_{i}-1\right) \epsilon_{\mathrm{C}}$ & & $\left(1-2 k_{i}\right) \epsilon$ \\
\hline 6 & - & + & - & $-k_{i} \epsilon_{A}$ & & $-k_{i} \epsilon$ \\
\hline 7 & & - & 1 & $-\left(k_{i}-1\right) \epsilon_{\mathrm{c}}$ & & $\left(1-k_{i}\right) \epsilon$ \\
\hline 8 & - & - & - & No UV absorption & & \\
\hline
\end{tabular}

absorptivities $\epsilon$, eight different cases can be distinguished as indicated in Table 1. In Table 1 the values of $K_{i}$ are given for all combinations of the ionic components $i, \mathrm{~A}$ and $\mathrm{C}$ if they are $\mathrm{UV}$ absorbing $(+)$ or are UV transparent $(-)$. The concepts usable in UV detection of "direct UV mode" and "indirect UV mode", whereby generally a BGE is chosen without any UV-absorbing properties or a BGE the co-ion of which has UV-absorbing properties respectively, correspond to cases 4 and 6 in Table 1, respectively. However, we shall use the term "direct UV mode" for cases 1-4 where the sample component has UV-absorbing properties and the term "indirect UV mode" for cases 5-7 where the sample component is UV transparent and these modes are given in the columns "UV mode" and "indirect UV mode". Case 8 is not useful, because no UV signal will be observed.

\subsection{Optimization in $U V$ detection}

From Table 1, it is clear that there are several ways to optimize the UV signal. In all cases in Table 1 an optimum UV response (optimum $K_{i}$ ) value can be obtained for minimum $k_{i}$ values or very large $k_{i}$ values, except for case $4\left(K_{i}\right.$ is always $\epsilon$ ) and case 6 where a maximum (negative) $K_{i}$ value is obtained for a maximum $k_{i}$ value. This means that the crucial point in the optimization of the absorbance $A$ is the value of
$K_{i}$ and by this maximizing or minimizing the value of $k_{i}$. To give an indication of the values of $k_{i}$, in Fig. 1 calculated lines of constant $k_{i}$ values are given, corresponding to sets of $m_{\mathrm{A}}$ and $m_{\mathrm{C}}$ values and a constant value for a sample ionic mobility $m_{i}$ of $40 \cdot 10^{-9} \mathrm{~m}^{2} / \mathrm{V} \cdot \mathrm{s}$. The arrows indicate increasing $k_{i}$ values. From Fig. 1 (and Eq. 4), it can be seen that for $m_{i}=m_{\mathrm{A}}$, the $k_{i}$ values and therefore the transfer ratios are always unity. Minimum $k_{i}$ values are obtained for a minimum mobility of the $A$ ions, and maximum $k_{i}$ values for a maximum mobility of the $\mathrm{A}$ ions, both at a maximum mobility of the $\mathrm{C}$ ions. It should be noted that minimum $k_{i}$ values, obtained for $m_{i}>m_{\mathrm{A}}$, mean that one ion of the BGE is displaced by more than one sample ion, through which the total ionic strength increases.

\subsection{Peaks or dips?}

The question of whether components can be detected as peaks or dips is answered by the values of $K_{i}$ given in Table 1. For positive values of $K_{i}$ positive peaks and for negative values of $K_{i}$ negative peaks (dips) are obtained. For an impression of the size of the UV signals in the different modes of UV detection, in Fig. 2 the calculated parameter $K_{i} / \epsilon$ is given for all $(\mathrm{A}, \mathrm{C})$ direct $U V$ modes and $(B, D)$ indirect $U V$ modes for varying mobilities of the $(A, B)$ co-ions $A$ and of $(\mathrm{C}, \mathrm{D})$ the sample ions $i$. All other 


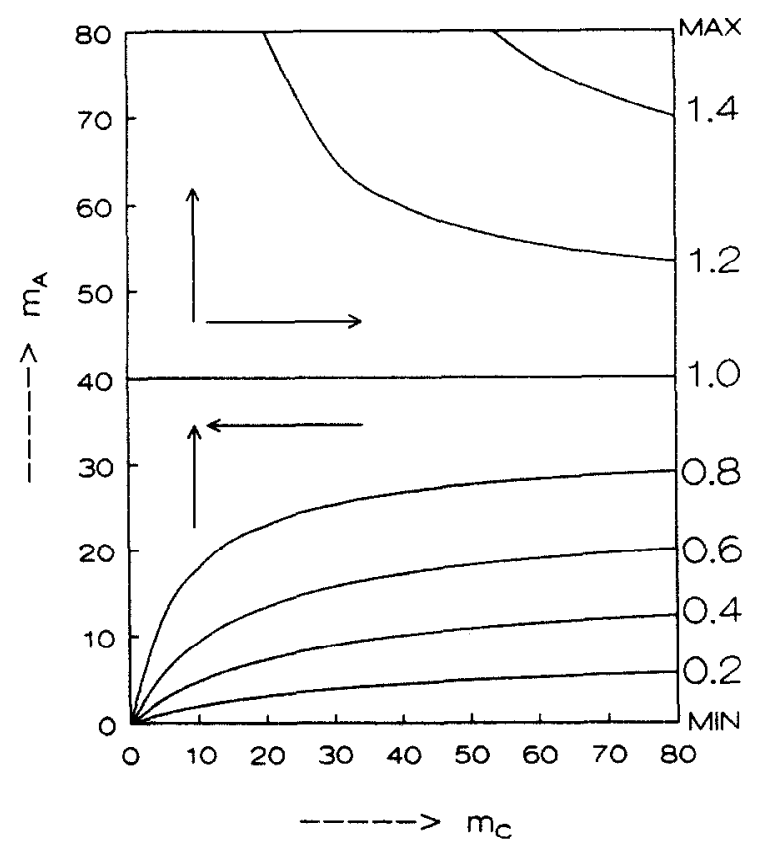

Fig. 1. Calculated lines of constant $k_{i}$ values for various mobilities of the co-ions $m_{\mathrm{A}}$ and of the counter ions $m_{\mathrm{C}}$ for a constant mobility of the sample ionic species of 40 . All mobilities are given in $10^{-9} \mathrm{~m}^{2} / \mathrm{V} \cdot \mathrm{s}$. The lines are indicated with numbers refering to the constant $k_{i}$ values of the corresponding lines. Maximum and minimum values of $k_{i}$ are obtained for a maximum and minimum value of $m_{\mathrm{A}}$, respectively, in combination with a maximum mobility of the counter ions $\mathrm{C}$. The arrows indicate increasing $k_{i}$ values.

mobilities are always taken as $40 \cdot 10^{-9} \mathrm{~m}^{2} / \mathrm{V} \cdot \mathrm{s}$. From Table 1 and Fig. 2, some interesting conclusions can be drawn. For the conventional direct UV mode, case 4 , the $K_{i} / \epsilon$ value is always 1 (see Fig. 2A and C). For cases 1 and 2, positive peaks are obtained if $m_{i}>m_{\mathrm{A}}$. Negative peaks (dips) are obtained, however, if $m_{i}<m_{\mathrm{A}}$. This means that the UV signal, although the sample and co-ion are assumed to have the same molar absorptivity, can be positive or negative depending on the mobility of the sample component. The explanation is simple. If the sample ionic mobility is lower than that of the co-ions, the response factor is larger than one through which the total ionic strength decreases, and although both ionic species are UV absorbing, the net UV signal decreases. If $m_{i}=m_{\mathrm{A}}$ the transfer ratio is just one and the UV signal is constant. For case
3, negative peaks are obtained for very low mobilitics of the sample ionic species.

If $m_{i}>m_{\mathrm{A}}$ the application of UV-absorbing counter-ions $\left(K_{i} / \epsilon=2-k_{i}\right)$ gives the best results. For the conventional indirect UV mode, case 6, and for case 5 (see Fig. 2B and D) generally negative UV peaks will be obtained. Positive peaks can, however, be obtained applying UV-absorbing counter ions $\mathrm{C}$, case $7\left(K_{i} / \epsilon=\right.$ $1-k_{i}$ ), for $m_{i}>m_{\mathrm{A}}$. Further, it is always favourable to apply both a UV-absorbing counter- and co-ion $\left(K_{i} / \varepsilon=1-2 k_{i}\right)$ for $m_{i}<m_{\mathrm{A}}$.

\section{Experimental}

For all CZE experiments a P/ACE System 2000 HPCE system (Beckman, Palo Alto, CA, USA) was used. All experiments were carried out with a Beckman eCAP capillary tubing (75 $\mu \mathrm{m}$ I.D.) with a total length $46.7 \mathrm{~cm}$ and a distance between injection and detection of 40.0 $\mathrm{cm}$. The wavelength of the UV detector was set at $214 \mathrm{~nm}$. All experiments were carried out in the cationic mode (anode at the inlet) and the operating temperature was $25^{\circ} \mathrm{C}$. Sample introduction was performed by applying pressure injection, where a 1-s pressure injection represents an injected amount of ca. $6 \mathrm{nl}$ and an injected length of $0.136 \mathrm{~cm}$. Data analysis was performed using the laboratory-written data analysis program CAESAR.

\section{Results and discussion}

In the theoretical part, the different indirect and direct UV modes have already been pointed out, and assuming an equal molar absorptivity for UV-absorbing components, eight different cases could be distinguished. The $K_{i} / \varepsilon$ values in Fig. 2 are a measure of the UV signal. In practice, molar absorptivities are different for the different components, through which both the $k_{i}$, determined by the mobilities of all ionic species, and the $\epsilon$ values of all ionic species play a role in the size of the UV signal.

For a demonstration of the different effects 

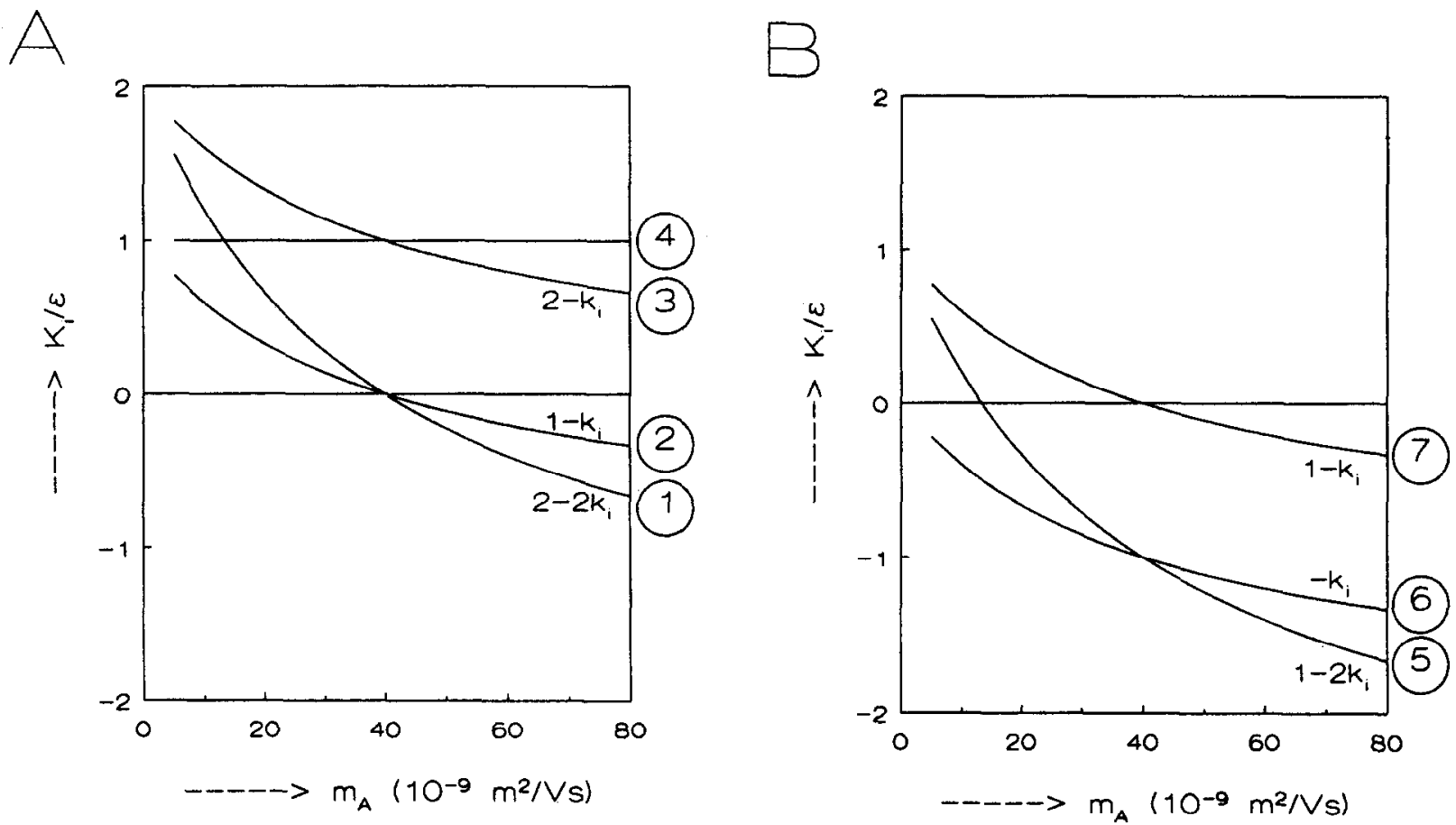

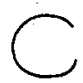
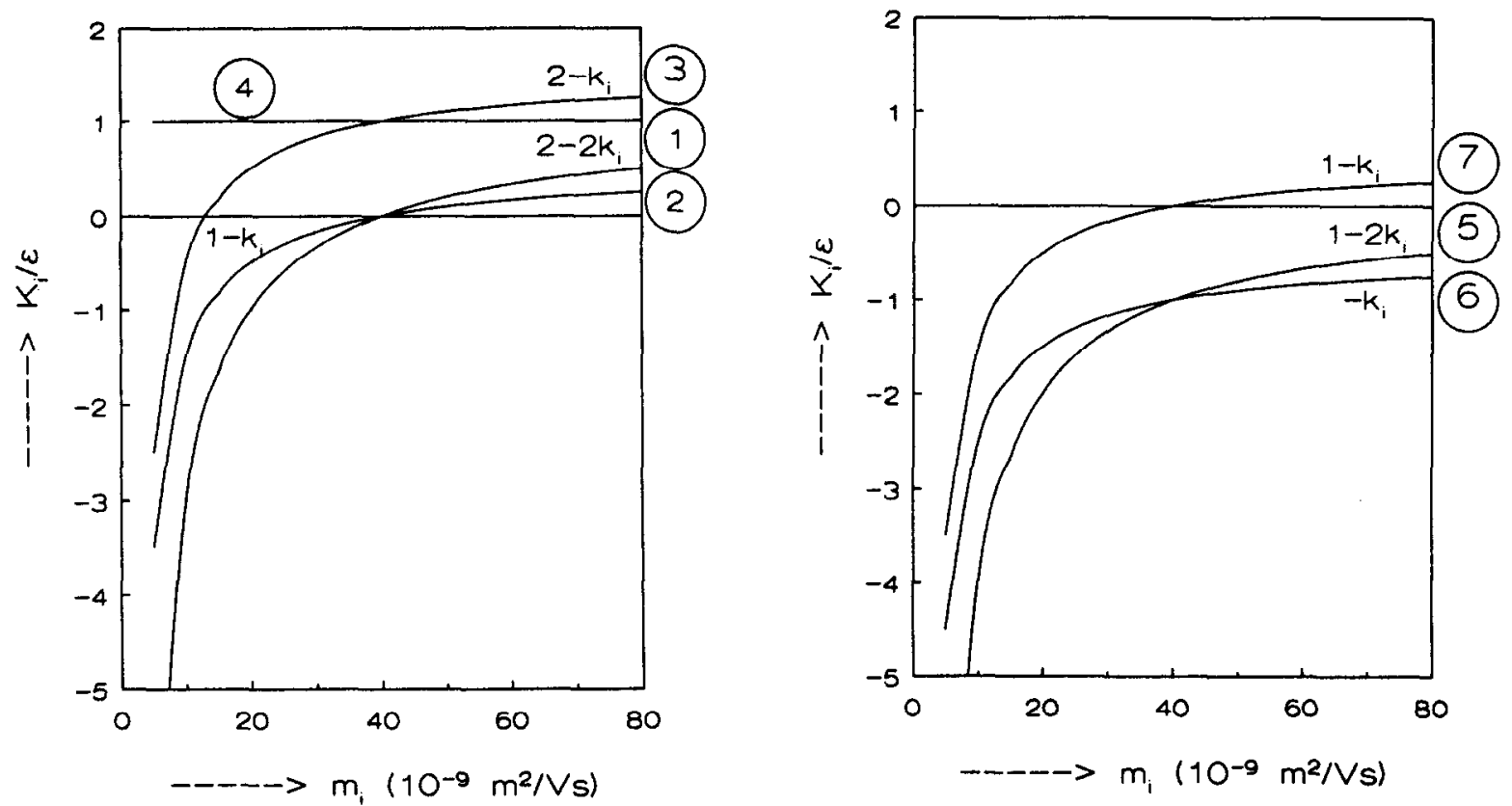

Fig. 2. Calculated values for the parameter $K_{i} / \epsilon$, assuming an equal molar absorptivity $\epsilon$ for all $U V$-absorbing components, for (A, C) direct UV modes and (B, D) indirect UV modes for various mobilities of the $(A, B)$ co-ions $A$ and of $(C, D)$ the sample ions $i$. All other mobilities are always taken as $40 \cdot 10^{-9} \mathrm{~m}^{2} / \mathrm{V} \cdot \mathrm{s}$. The lines are indicated with encircled numbers refering to the cases in Table 1 and with the values of $K_{i} / \epsilon$. For further information see text and Table 1. 
playing a part in the UV detection, an equimolar solution of five UV-transparent cations, potassium, sodium, tetramethylammonium (TMA), tetracthylamonium (TEA) and tetrabutylammonium (TBA), is used, which will be denoted as the MIX. In the electropherograms, peaks for the different components of the MIX are indicated with the numbers $1,2,3,4$ and 5 for the ions potassium, sodium, TMA, TEA and TBA, respectively. In Fig. 3 the electropherograms are given for the separation of 5-s pressure injections of $5 \cdot 10^{-4} M$ of the MIX applying BGEs containing UV-absorbing co-ions (case 6) and containing both UV-absorbing counter and co-ions (case 5). The BGEs containing UV-absorbing co-ions consisted of $0.01 M$ histidine (HiAc) and $0.01 M$ imidazole ( $\operatorname{ImAc}$ ), respectively, adjusted to $\mathrm{pH} 4.2$ by adding acetic acid. For all BGEs, histidine or imidazole was chosen as the UVabsorbing co-ion because their $\mathrm{p} K$ values are ca. 6 and 7, respectively, through which these cat-

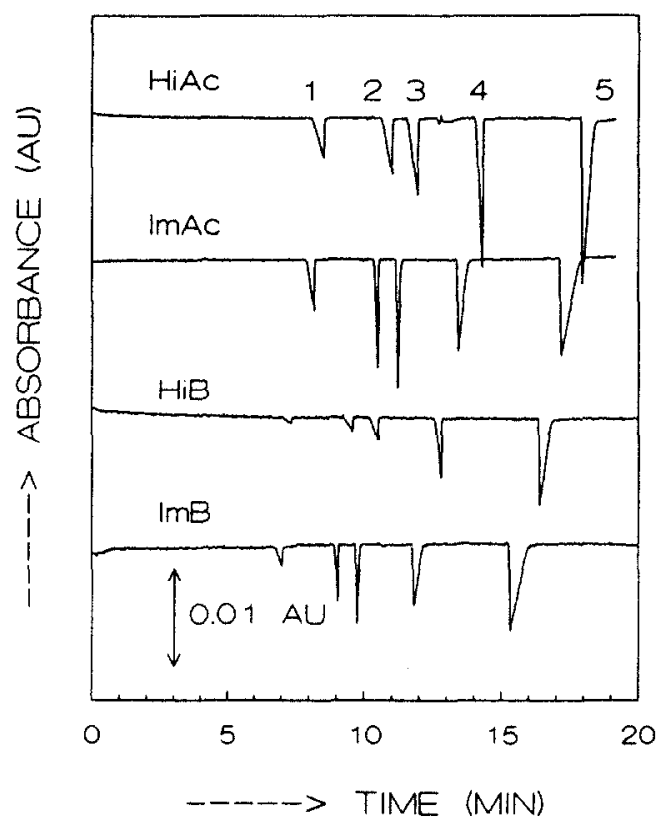

Fig. 3. Electropherograms for the separation of 5-s pressure injections of $5 \cdot 10^{-4} M$ of the MIX applying BGEs of histidine acetate (HiAc), imidazole acetate (ImAc), histidine benzoate ( $\mathrm{HiB})$ and imidazole benzoate ( $\mathrm{ImB}$ ). All BGEs consisted of $0.01 M$ of the cations, adjusted to $\mathrm{pH} 4.2$ by adding the buffering acids. The applied voltage was $5 \mathrm{kV}$. For further information, see text. ions are protonated for the larger part at the working $\mathrm{pH}$. In the case of $\mathrm{HiAc}$, the mobility of histidine is lower than that of imidazole, the last peaks on the electropherogram are larger and more symmetrical, whereas in the BGE ImAc the first peaks are larger, although the molar absorptivity of imidazole is only about two thirds of that of histidine. This corresponds to the guideline that the mobility of the co-ions of the BGE must be as close as possible to that of the sample component, in order to diminish the effect of electrodispersion. For BGEs with both UV-absorbing counter and co-ions, we adjusted $0.01 M$ solutions of histidine (HiB) and imidazole $(\operatorname{ImB})$ to $\mathrm{pH} 4.2$ by adding benzoic acid. Although the molar absorptivities of histidine, imidazole and benzoic acid are not equal, so that Fig. 2D is not valid, we can see the effect that by addition of UV-absorbing counter ions to the BGE, all peaks for components with high mobilities are much smaller than those in the corresponding BGE with only UV-absorbing coions. This can easily be understood. The transfer ratio is smaller than unity for components with a high mobility through which the total ionic strength increases and therefore also the concentration of the UV-absorbing counter ions increases, counteracting the decrease in UV signal by the lower concentration of the co-ions. For the system $\mathrm{HiB}$ the resulting peaks for potassium and sodium are very small. This effect is smaller in the system $\operatorname{ImB}$ because of the higher mobility of imidazole.

In Fig. 4 the relationship between calculated $K_{i} / \epsilon$ values and the mobilities of sample components are given for BGEs with UV-absorbing counter ions (case 7), viz., $0.01 M$ Tris, $0.01 M$ lithium and $0.01 M$ potassium ions adjusted to $\mathrm{pH} 4.2$ by adding benzoic acid. In the calculations no corrections are made for activities and relaxation and electrophoretic effect. Because all ionic species are monovalent, changes will be in the same order of attitude for all ionic species. Just as expected according to Fig. 2D, positive peaks can be expected for sample components with $m_{i}>m_{\mathrm{A}}$. The positions of the components of the MIX are indicated with the dotted lines in Fig. 4. 


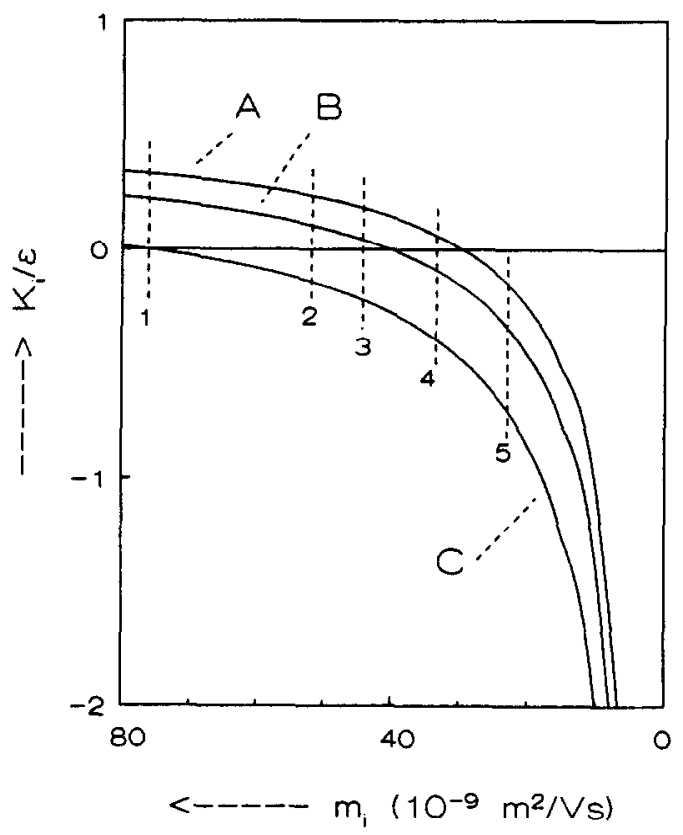

Fig. 4. Relationship between calculated $K_{i} / \epsilon$ values and the mobilities of sample components for BGEs with UV-absorbing counter ions (case 7), (A) $0.01 \mathrm{M}$ Tris-, (B) $0.01 \mathrm{M}$ lithium- and (C) $0.01 M$ potassium-benzoate at $\mathrm{pH}$ 4.2. The dotted lines indicate the values of $K_{i} / \epsilon$ of the cations of the MIX on the curves.

In Fig. 5 the electropherograms are given for the separations of 5 -s pressure injections of 5 . $10^{-4} M$ of the MIX applying (A) $0.01 M$ Trisbenzoate, (B) $0.01 M$ lithium-benzoate and (C) $0.01 M$ potassium-benzoate. The applied voltage was $5 \mathrm{kV}$. According to the theory (see Fig. 4), potassium ions are not visible in system $C$, whereas all components are dips. In system B the first three components are peaks and the last two are dips, whereas in system A only the last component is a dip, in accordance with the positions indicated in Fig. 4.

\subsection{System peaks in $C Z E$}

Beckers [11] described that moving boundary zones can originate from discontinuities in the concentration of the co-ions and/or $\mathrm{pH}$ of the $\mathrm{BGE}$ and showed that injected block-shaped discontinuities in concentration and/or $\mathrm{pH}$ of the BGE split up in a migrating part with a mobility

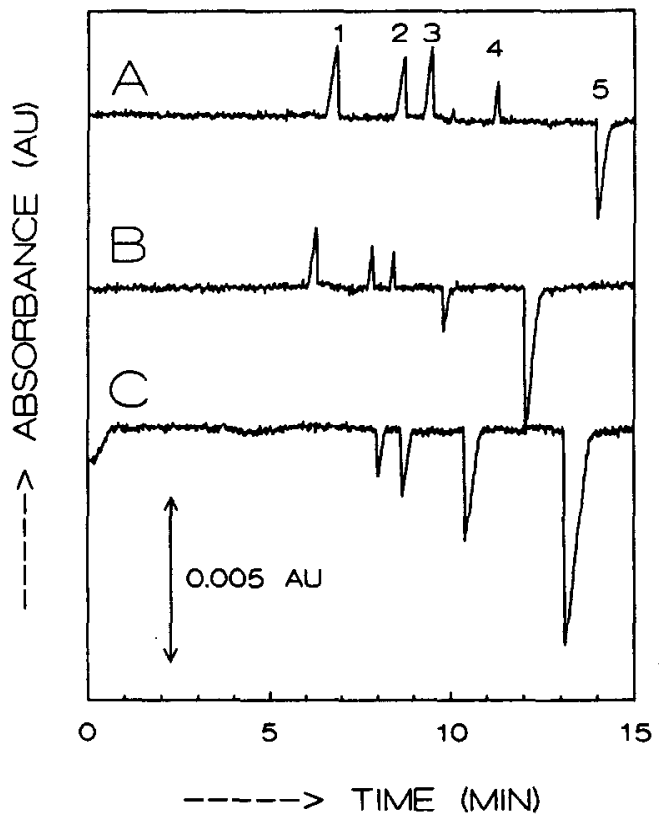

Fig. 5. Electropherograms for the separations of 5-s pressure injections of $5 \cdot 10^{-4} M$ of the MIX applying (A) $0.01 M$ Tris-benzoate, (B) $0.01 M$ lithium-benzoate and (C) $0.01 M$ potassium-benzoate at $\mathrm{pH} 4.2$. Applied voltage, $5 \mathrm{kV}$.

determined by the composition of the BGE and a part migrating with the velocity of the electroosmotic flow (EOF) at the position of the original disturbance. System peaks are the result. Although the system peak migrating with the velocity of the EOF is at the same position as system peaks described by De Bruin et al. [12], we think that the natures of these system peaks are different. The system peak described by De Bruin et al. are a result of the adaptation to the value of the local Kohlrausch function $\omega$, whereas the system peaks described by Beckers [11] are also dependent on discontinuities in, e.g., $\mathrm{pH}$ through which Kohlrausch's regulation function is not constant. The discontinuities are only observable for UV-absorbing BGEs. The concentration of the UV-absorbing co-ions in a system peak can be higher or lower than that of the BGE and we shall denote these system peaks as "positive" or "negative" peaks, respectively. Further, the local electric field strength $E_{\mathrm{SP}}$ can be higher or lower than that of the $\mathrm{BGE}, E_{\mathrm{BGE}}$, depending on the mobilities and concentrations 
of all ionic species concerned. If $E_{\mathrm{SP}}<E_{\mathrm{BGE}}$ the peaks are diffuse at the front-side and for $E_{\mathrm{SP}}>$ $E_{\mathrm{BGE}}$, as in ITP, they are sharp at the front-side.

All parameters, such as the mobility of such a system peak, the concentration of all ionic species and the $\mathrm{pH}$ in the system peak, can be calculated with a mathematical model [11]. In Fig. 6 the four types of system peak shapes are shown schematically. Positive peaks can be of the type $\mathrm{A}$ or $\mathrm{C}$, respectively sharp and diffuse at the front-side, and negative peaks of the type $B$ or $\mathrm{D}$, respectively diffuse and sharp at the frontside. For a smaller system peak, i.e., a system peak with a concentration of the co-ion closer to that of the BGE, the peak shape is given by dotted lines. For a specific $\mathrm{BGE}$ composition generally the pairs $\mathrm{A}-\mathrm{B}$ or $\mathrm{C}-\mathrm{D}$ are obtained as system peaks. Whether the positive or negative system peak appears depends to the kind of discontinuity introduced.

As an illustration, in Fig. 7 the electropherograms are given for the separation of a 5-s

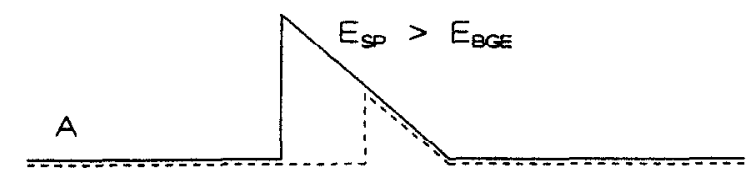

$$
\mathrm{B}
$$

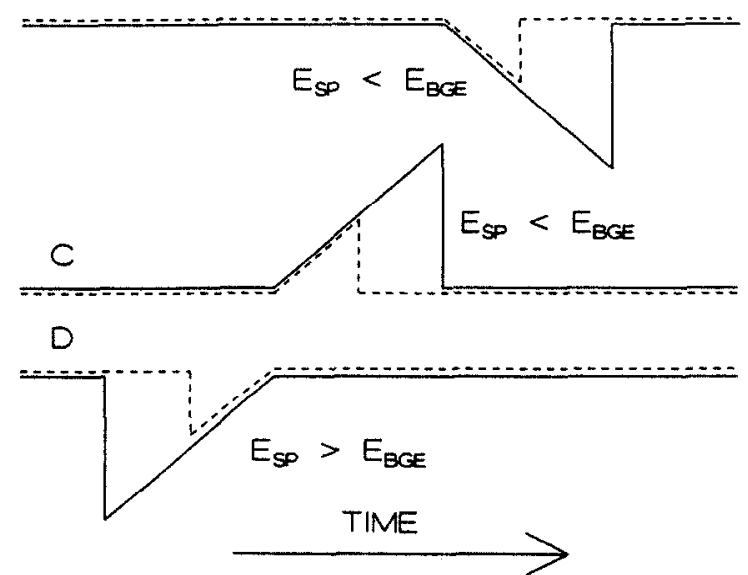

Fig. 6. Schematic representation of four types of system peak (SP) shapes. Positive peaks can be of type A or C, respectively sharp or diffuse at the front-side, and negative peaks of the type $B$ or $D$, respectively diffuse and sharp at the front-side. For smaller system peaks the peak shape is given as dotted lines. For further information, see text.

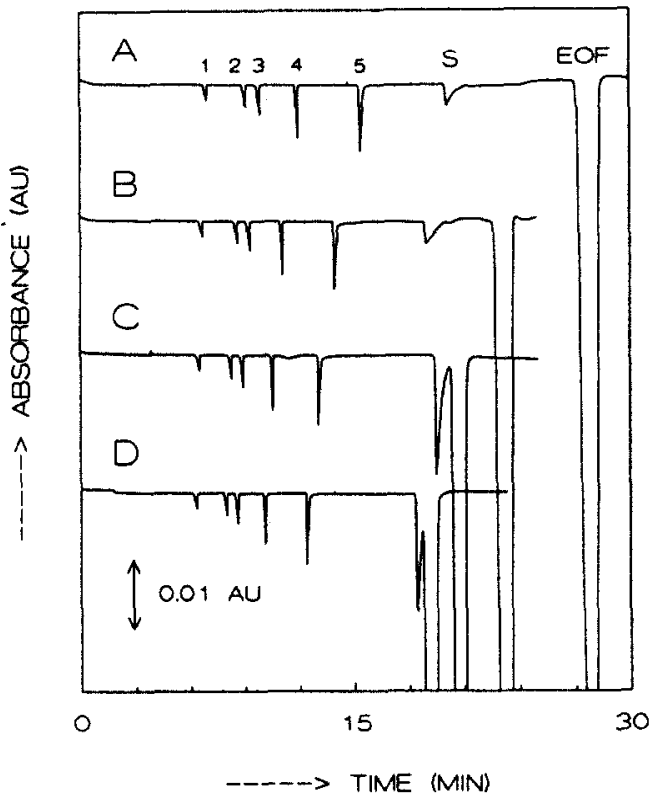

Fig. 7. Electropherograms for the separation of a 5-s pressure injection of $0.0002 \mathrm{M}$ of the MIX on applying BGEs consisting of $0.01 M$ histidine adjusted to a $\mathrm{pH}$ of (A) 4.0 and (B) 4.5 by adding formic acid and (C) 4.5 and (D) 5 by adding acetic acid. The mobilities of the system peaks $S$ are higher for lower system pHs. Applied voltage, $5 \mathrm{kV}$.

pressure injection of $0.0002 M$ of the MIX on applying BGEs consisting of $0.01 M$ histidine adjusted to a pH of (A) 4.0 and (B) 4.5 by adding formic acid and (C) 4.5 and (D) 5 by adding acetic acid. Clearly a system peak of type $D$ can be seen in all instances. The mobility of the system peaks is larger for lower system pHs.

\subsection{BGE with two co-ions}

Often, BGEs consisting of more than one coion are applied. For example, De Bruin et al. [12] added polassium chloride to the BGE to determine the effect of the ionic strength on sensitivity and efficiency. Sometimes two co-ions are used, where one has UV-absorbing properties whereas the other acts as buffer or complexing ions. On applying BGEs with two coions, system peaks, with a different character as discussed above (see Fig. 7), often occur. It is remarkable that the mobilities of the sample components determine whether a positive or 
negative system peak occurs and the mobilities of the system peaks determine whether a positive or negative component peak is present for the UV-transparent components.

In all our experiments it appears that if the mobility of a component was higher than that of the system peak S, the UV-transparent component gave a positive peak. If the mobility was lower, a negative peak was the result. System peaks belonging to components with a mobility higher than that of the system peak are negative by themselves, otherwise they are positive. As an illustration, in Fig. 8 the electropherograms are given on applying a BGE consisting of 0.004 $M$ histidine and $0.006 M$ potassium ions adjusted to $\mathrm{pH} 5$ by adding acetic acid for 5-s pressure injections of $0.0005 \mathrm{M}$ solutions of the ions (B)

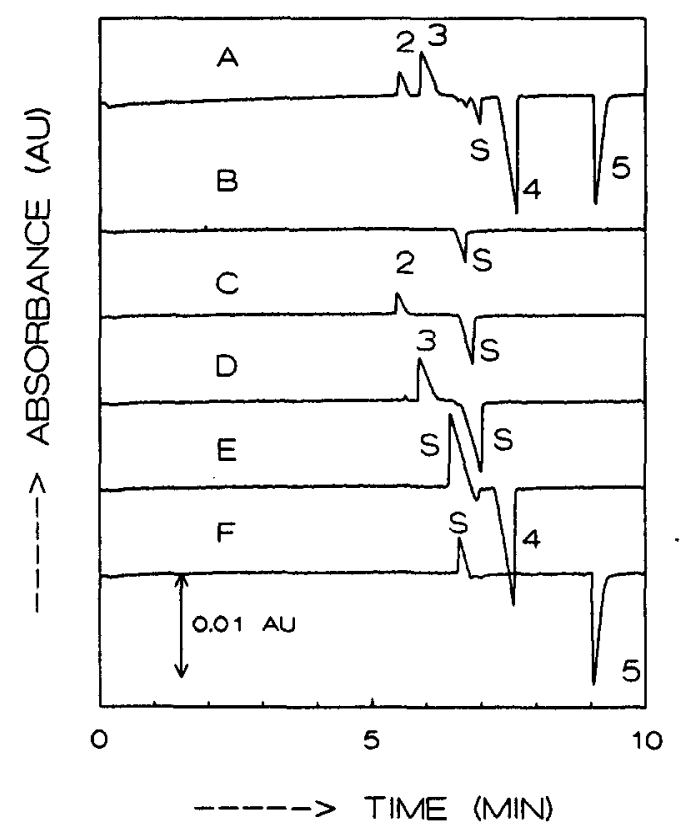

Fig. 8. Electropherograms for the separations of 5-s pressure injections of $0.0005 \mathrm{M}$ solutions of the ions (B) potassium, (C) sodium, (D) TMA, (E) TEA and (F) TBA and (A) a mixture of these components on applying a BGE consisting of a mixture of $0.004 \mathrm{M}$ histidine and $0.006 \mathrm{M}$ potassium ions adjusted to $\mathrm{pH} 5$ by adding acetic acid. The applied voltage was $7 \mathrm{kV}$. If the mobilities of the sample components are higher than that of the system peak $S$, the sample components are positive peaks, otherwise they are negative. If the system peak is in the vicinity of a sample component, both peaks are enlarged. potassium, (C) sodium, (D) TMA, (E) TEA and (F) TBA and (A) a mixture of these components. The applied voltage was $7 \mathrm{kV}$. Although in all the electropherograms two system peaks were present, the EOF dip and the system peak just before the EOF dip are not shown. In Fig. $8 \mathrm{~B}$ potassium is not visible, because the $\mathrm{BGE}$ contains potassium. As its mobility is higher than that of the system peak, the system peak $S$ is negative. The mobilities of $(C)$ sodium and (D) TMA are higher than that of the system peak $S$, hence these components show a positive component peak, whereas the system peaks S belonging to them are negative. For (E) TEA and (F) TBA the mobilities are lower than that of the system peak and therefore the component peaks are negative and the system peaks $S$ are positive.

If a system peak and the component peak are close together there seems to be an interaction between the two peaks. Both peaks are enlarged, as if the first peak sucks empty the second peak. Remember that a positive peak means a concentration of UV-absorbing co-ion higher than that in the BGE, whereas in a dip this concentration is lower than that in the BGE. In (A) the electropherogram for the separation of all components is given. Sodium (2) and TMA (3) are positive peaks and TEA (4) and TBA (5) are negative peaks. The resulting system peak $S$ is a negative peak. The type of the system peaks $S$ depends on the mobilities of the sample components injected and are of the $A-B$ type from Fig. 6.

In Fig. 9A the relationship between the measured mobilities of the system peaks $S$ and the concentrations of potassium ions is given for BGEs consisting of the co-ions potassium and histidine at a total cationic concentration of 0.01 $M$ adjusted to a $\mathrm{pH}$ of 5 by adding acetic acid. Because the values of the mobilities of system peaks can very considerably, as this mobility is determined from a large positive peak or negative system peak (see Figs. 6 and 8 ), we calculated the mobility of the system peak $S$ as the average from a positive and negative system peak. It is remarkable that in Fig. 9 the mobilities of the system peaks seem to vary between 


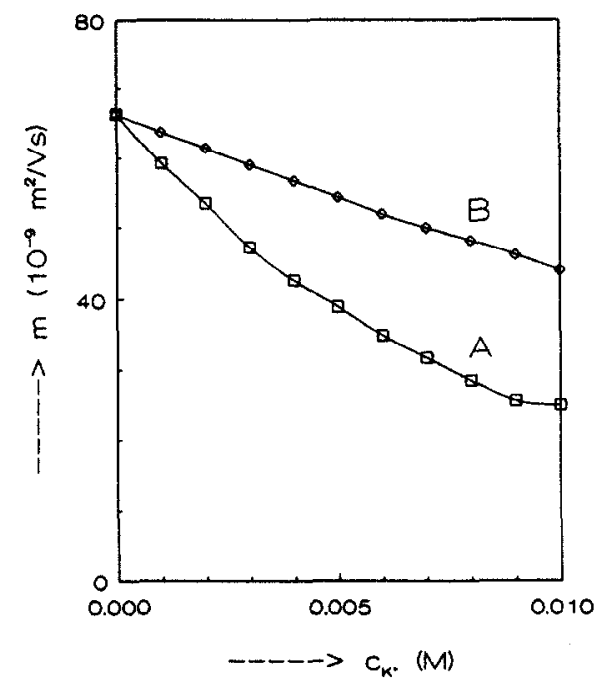

Fig. 9. Relationship between the measured mobilities of the system peaks $S$ and the concentrations of potassium ions for BGEs consisting of the co-ions (A) potassium and histidine and (B) potassium and imidazole at a total cationic concentration of $0.01 \mathrm{M}$ adjusted to $\mathrm{pH} 5$ by adding acetic acid. The mobilities indicated at $c_{\mathrm{K}^{+}}$values of 0.000 and $0.010 \mathrm{M}$ are the measured mobilities of the potassium and imidazole and histidine ions, respectively.

the mobility of the potassium ions and that of the histidine ions. Therefore, we measured again the mobilities of the system peaks for BGEs consisting of mixtures of potassium and imidazole at a total cationic concentration of $0.01 \mathrm{M}$ and at a $\mathrm{pH} 5$ by adding acetic acid. The results are given in Fig. 9B. Again the mobilities of the system peaks vary between the mobility of imidazole and potassium ions.

To give a complete survey of all the foregoing effects for different compositions of the $\mathrm{BGE}$, in Fig. 10 the electropherograms are given for the separations of 5 -s pressure injections of $0.0005 \mathrm{M}$ of the MIX applying BGEs consisting of varying concentrations of potassium and histidine at a total cationic concentration of $0.01 M$ and adjusted to $\mathrm{pH} 5$ by adding acetic acid. The electropherograms are indicated with a number referring to the concentration of the histidine ion is in the BGE. Applying the BGE with $0.01 M$ histidine, the components potassium (1), sodium (2), TMA (3), TEA (4) and TBA (5) are all visible as negative peaks in the indirect $\mathrm{UV}$

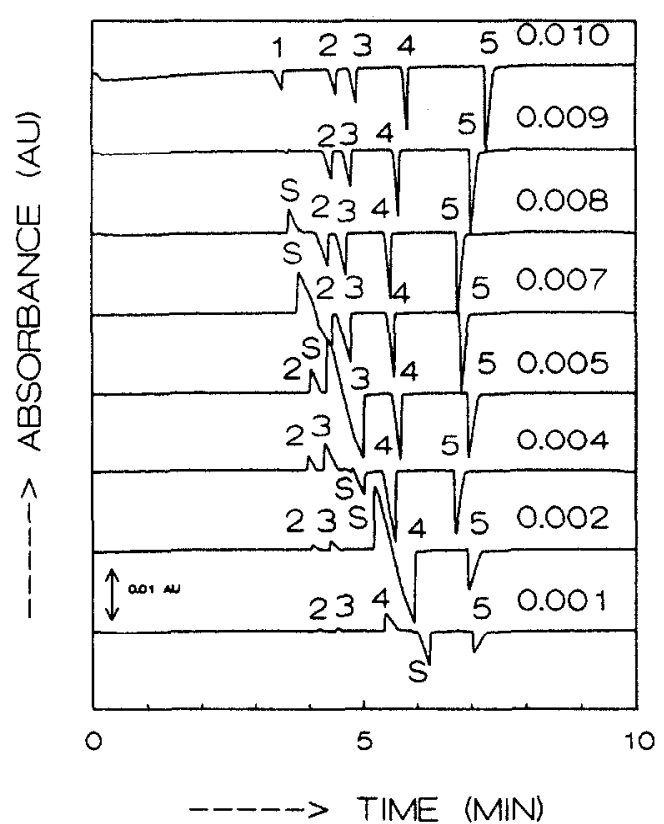

Fig. 10. Electropherograms for the separation of 5-s pressure injections of a mixture of $0.0005 M$ of the MIX applying BGEs consisting of varying concentrations of potassium and histidine at a total cationic concentration of $0.01 \mathrm{M}$, adjusted to $\mathrm{pH} 5$ by adding acetic acid. The electropherograms are indicated with a number refering to the concentration of the histidine ions in the BGE. The applied voltage was $7 \mathrm{kV}$. The mobility of the system peak $S$ varies considerably. Components with mobilities higher than that of the system peaks are peaks and components with lower mobilities are dips.

mode. In the BGE with $0.009 M$ histidine (and $0.001 M$ potassium) the potassium peak is not visible because the BGE contains potassium. A small dip marks the position of potassium. In the BGE with $0.008 M$ histidine a system peak $\mathrm{S}$ is present. Because the mobility of the system peak $S$ is higher than that of any sample component, all sample peaks are negative and the system peak is a positive peak. In the BGE with 0.007 $M$ histidine the system peak and the sodium peak are very close, hence these peaks are enlarged. In the BGE with $0.005 M$ histidine the system peak passed the sodium peak, so sodium is a positive peak. The system peak is still a positive peak and because it is close to the TMA peak, these peaks are enlarged. In the BGE with $0.004 M$ histidine the system peak passed the TMA peak and lies between TMA and TEA as a 
negative peak. Sodium and TMA are positive peaks and TEA and TBA are still negative peaks. In the BGE with $0.002 M$ histidine the system peak $S$ and TEA are close together and both enlarged. It is not clear which peak is the system peak and TEA. In the BGE with $0.001 M$ histidine the system peaks passed TEA and is a negative peak between TEA and TBA. Sodium, TMA and TEA are positive peaks, small owing to low concentration of histidine, whereas the system peak $S$ and TBA are negative peaks. In all cases were measured all components separately to identify carefully all peaks, although it is difficult to identify the peaks if a system peak and a component peak are close together.

\subsection{Calibration graphs in BGEs with two co-ions}

As already indicated, system peaks appear on applying BGEs with two co-ions and their mobilities are between the mobilities of the two co-ions. In all systems measured, components with a mobility higher than that of the system peak are positive peaks whereas the system peaks themselves are negative, and vice versa. Further, if the system peak and component peak are close together, both peaks are enlarged and the quantitative properties seems to be lost.

To study this effect, calibration graphs were measured by injecting $0.0002 M$ of the MIX for several pressure injection times applying a BGE consisting of $0.005 M$ histidine and $0.005 M$ potassium adjusted at $\mathrm{pH} 5$ by adding acetic acid. The applied voltage was $5 \mathrm{kV}$. As can be seen in Fig. 10, sodium and the system peak are positive, whereas TMA, TEA and TBA are negative peaks. The system peak $S$ and the peak of TMA are close together and enlarged. Compared with several other BGEs the TMA peak is generally smaller than those of the TEA and TBA peaks.

In Fig. 11 the relationship between measured peak area (mAUs) and pressure injection times (s) of $0.0002 M$ of the MIX is given for all components. As can be seen, there is linear relationship between peak area and pressure injection time for sodium (positive peaks) and

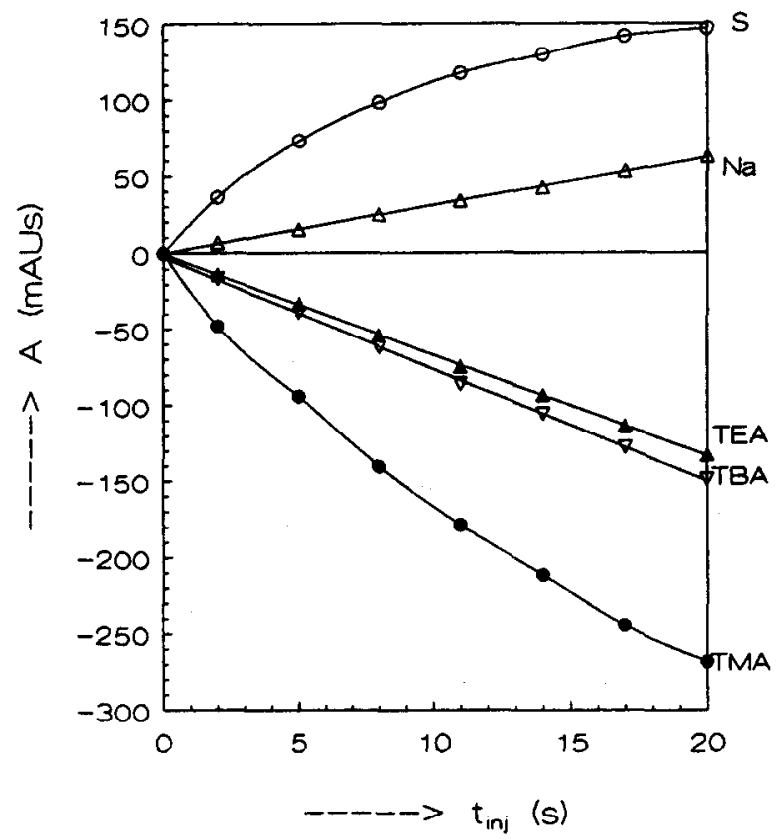

Fig. 11. Relationship between measured peak area $\mathbf{A}$ (mAUs) and pressure injection times (s) of $0.0002 M$ of the MIX applying a BGE of $0.005 M$ potassium and $0.005 M$ histidine adjusted to $\mathrm{pH} 5$ by adding acetic acid. The applied voltage was $7 \mathrm{kV}$. A linear relationship between peak arca and pressure injection times is obtained for sodium (positive peaks) and TEA and TBA (negative peaks) with regression correlation coefficients larger than 0.999 . For the system peak $S$ and the TMA peak the linear relationship is lost and these peaks are much enlarged.

TEA and TBA (negative peaks) with regression correlation coefficients larger than 0.999 . For the system peak $S$ and TMA peak the linear relationship is lost and these peaks are much enlarged.

\section{Conclusion}

Starting from Kohlrausch's law, derived for fully ionized monovalent ionic constituents, an expression for the response factor can be derived showing that the response factor is small if the mobility of the sample ionic species is higher than that of the co-ion of the BGE. In that case the total ionic strength in the sample zone increases. On applying UV-absorbing counter ions, the total UV absorbance can increase, even 
if the sample components are UV transparent. UV-transparent components can show positive peaks in this way. For sample components with very low mobilities the response factor can be very large. If the BGE has UV-absorbing properties, the net UV signal is lower and even UV-absorbing components show dips in such cases. If BGEs are applied with two co-ions, system peaks are the result. This means that the ratio of the concentrations of the two co-ions is not constant in the electrolyte system. The mobility of the system peak is between those of the co-ions and is dependent on the ratio of the concentrations of the co-ions. For BGEs containing potassium and histidine as co-ions, UVtransparent sample components with a mobility higher than that of the system peak appear as peaks, whereas UV-transparent sample components with lower mobilities are dips. Also, the system peaks themselves can be positive peaks or dips. If the sample component is a peak, the system peak is a dip and vice versa. For complex sample mixtures the system peak is a peak or a dip dependent on the sample composition. If the peaks of a system peak and a sample component are close together, they interact, resulting in an enlarged peak and dip, through which quantitative aspects are lost.

Denkert et al. [13] reported that sometimes samples give positive or negative peaks depending on their charge and retention relative to the UV-absorbing ionic component in the mobile phase in reversed-phase ion-pair chromatography, and these phenomena seem to be in accord with phenomena described in this paper for CZE. To answer the question of whether these phenomena in CZE are associated with adsorption phenomena since the UV-absorbing ions are cations that can adsorb on the fuscdsilica surface producing system peaks just as in chromatography, experiments were carried out to separate anionic species by applying BGEs consisting of mixtures with various concentrations of a UV-absorbing and a UV-transparent anion. Also in the separation of anionic species, system peaks were present and if system peaks and sample components are close together they interact, resulting in enlarged peaks.
In Fig. 12 the electropherograms are given for the separation of 10-s pressure injections of a mixture of $2 \cdot 10^{-4} M$ propionic acid (P) and butyric acid (B) with BGEs consisting of mixtures of phenylacetic acid and acetic acid at various concentrations, (1) 0.01 and $0 \mathrm{M}$, (2) 0.008 and $0.002 \mathrm{M}$, (3) 0.004 and $0.006 \mathrm{M}$ and (4) 0.002 and $0.008 \mathrm{M}$, adjusted to $\mathrm{pH} 8.1$ by adding Tris. In electropherogram (1) applying only the UV-absorbing anion phenylacetic acid, propionic and butyric acids are present as a dip in the indirect UV mode. Applying a BGE with two co-ions a system peak $S$ appears. In electropherogram (2) the mobility of the system peak is higher than that of both anionic species and they are dips. In electropherogram (3) the system peak is in the vicinity of the peak of propionic

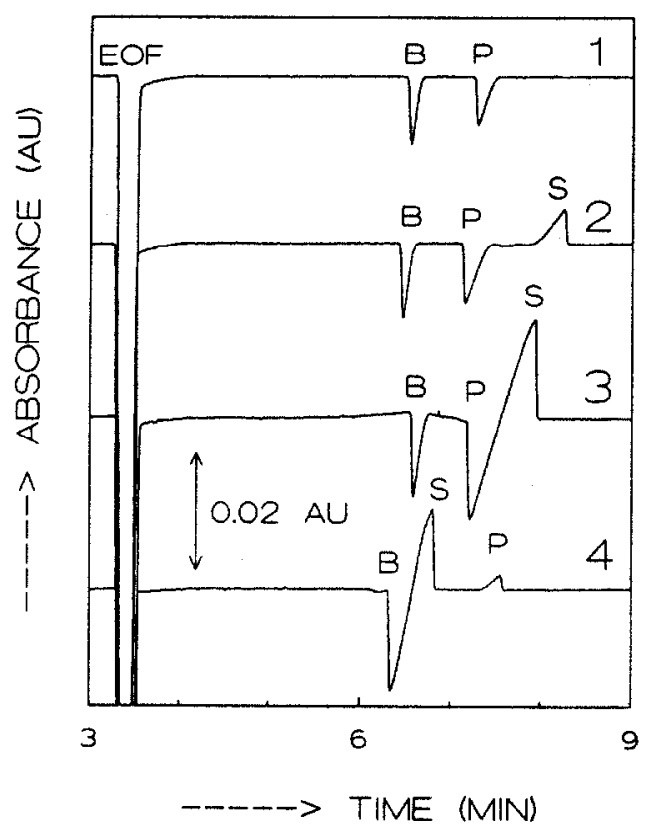

Fig. 12. Electropherograms for the separation of 10-s pressure injections of a mixture of $2 \cdot 10^{-4} M(\mathrm{P})$ propionic acid and (B) butytic acid applying BGEs consisting of mixtures of phenylacetic acid and acetic acid at concentrations of, respectively, (1) 0.01 and $0 M$, (2) 0.008 and $0.002 M$, (3) 0.004 and $0.006 M$ and (4) 0.002 and $0.008 M$ adjusted to $\mathrm{pH} 8.1$ by adding Tris. The applied voltage was $15 \mathrm{kV}$. Just as for cationic components, sample components with a mobility lower than that of the system peak are dips, whereas sample components with higher mobilities are peaks. For further explanation, see text. 
acid and both peaks are enlarged. In electropherogram (4) the system peak has a mobility lower than that of propionate, through which propionate is a peak whereas the butyrate and system peaks are enlarged since they interact together. The mobilities of the system peaks are between those of the anions of the BGE. From Fig. 12, it can be concluded that phenomena as described for cationic components also occur for anionic species, indicating that these phenomena and the presence of system peaks seems not to be associated with adsorption phenomena as in chromatography. The nature of thesc system peaks is under further investigation.

\section{Acknowledgement}

The author expresses his gratitude to Rian Straatman for her very skilful assistance in the experimental work.

\section{References}

[1] E.S. Yeung and W.G. Kuhr, Anal. Chem., 63 (1991) 275A.

[2] W.G. Kuhr and E.S. Yeung, Anal. Chem., 60 (1988) 1834.

[3] W.G. Kuhr and E.S. Yeung, Anal. Chem., 60 (1988) 2642.

[4] T. Wang and R.A. Hartwick, J. Chromatogr., 607 (1992) 119.

[5] M.W.F. Nielen, J. Chromatogr., 588 (1991) 321.

[6] F. Foret, S. Fanali, L. Ossicini and P. Bocek, $J$. Chromatogr., 470 (1989) 299.

[7] F. Kohlrausch, Ann. Phys. (Leipzig), 62 (1897) 209.

[8] F.E.P. Mikkers, Thesis, University of Technology, Eindhoven, 1980.

[9] F.E.P. Mikkers, Th.P.M. Verheggen and F.M. Everaerts, J. Chromatogr., 169 (1979) 1.

[10] M.T. Ackermans, F.M. Everaerts and J.L. Beckers, J. Chromatogr., 549 (1991) 345.

[11] J.L. Beckers, J. Chromatogr. A, 662 (1994) 153.

[12] G.J.M. de Bruin, A.C. van Asten, X. Xu and H. Poppe, J. Chromatogr., 608 (1992) 97.

[13] M. Denkert, L. Hackzell, G. Schill and E. Sjögren, $J$. Chromatogr., 218 (1981) 31. 